\title{
Regulation of differentiation in trabecular bone-derived mesenchymal stem cells by $T$ cell activation and inflammation
}

\author{
XINGHUO WU ${ }^{1 *}$, WENJUAN WANG $^{2 *}$, CHUNQING MENG $^{1}$, SHUHUA YANG ${ }^{1}$, \\ DEYU DUAN ${ }^{1}$, WEIHUA XU ${ }^{1}$, XIANZHE LIU ${ }^{1}$, MING TANG ${ }^{1}$ and HONG WANG ${ }^{1}$ \\ ${ }^{1}$ Department of Orthopaedics, Union Hospital, Tongji Medical College, Huazhong University of Science \\ and Technology, Hubei, Wuhan 430022; ${ }^{2}$ Department of Plastic Surgery, Tongji Hospital, Tongji Medical \\ College, Huazhong University of Science and Technology, Hubei, Wuhan 430030, P.R. China
}

Received April 29, 2013; Accepted June 4, 2013

DOI: $10.3892 / o r .2013 .2687$

\begin{abstract}
Mesenchymal stem cells (MSCs) are multipotent stem cells with the ability to migrate to sites of inflammation and injury, where they participate in tissue regeneration and repair. The present study aimed to investigate the effects of $\mathrm{T}$ cell activation and inflammation on the differentiation of MSCs. Human trabecular bone-derived MSCs were isolated from patients undergoing total hip replacement, and T cells were isolated and purified from peripheral blood mononuclear cells (PBMCs) using CD3 MicroBeads. MSCs were co-cultured with activated $\mathrm{T}$ cells to mimic the inflammatory microenvironment. MTS assay was used to detect cell proliferation. qRT-PCR, western blotting, histology and immunohistochemical staining were used to detect the adipo-/osteo-specific gene expression and the relative signaling pathway. The MTS results showed that higher concentrations of $\mathrm{T}$ cells significantly increased the proliferation of MSCs. Expression of the inflammatory gene IL-6 was upregulated, while expression of IL-10 and INF $\gamma$ was downregulated in MSCs exposed to activated T cells. The results also showed that PHA-activated $\mathrm{T}$ cells significantly upregulated the expression of PPAR $\gamma$ and FABP4 (adipo-specific genes) in MSCs, but no difference was noted in the expression of RUNX2, osteocalcin and ALP (osteo-specific genes) at the protein level. T cell treatment and inflammation inhibited the protein expression of TGF- $\beta 1$ and the phosphorylation of Smad3, resulting in the weakening of the TGF- $\beta /$ Smad pathway and enhancing the adipogenic differentiation of MSCs. The results indicated that PHA-activated
\end{abstract}

Correspondence to: Professor Hong Wang, Department of Orthopaedics, Union Hospital, Tongji Medical College, Huazhong University of Science and Technology, Hubei, Wuhan 430022, P.R. China

E-mail: wanghwh64@yahoo.cn

${ }^{*}$ Contributed equally

Key words: mesenchymal stem cells, T lymphocytes, inflammation, adipogenesis, osteogenesis
T cells and inflammation could promote adipogenesis without affecting the late stage of osteogenesis of MSCs, by increasing the expression of key adipogenic genes through TGF- $\beta / \mathrm{Smad} 3$ signaling.

\section{Introduction}

The most commonly used source of mesenchymal stem cells (MSCs) is bone marrow aspirate, but its disadvantages restrict clinical and laboratory practice, such as pain, morbidity and low cell number upon harvest. Recent studies have shown that MSCs can be obtained from trabecular bone fragments obtained during total hip/knee replacements, which can avoid the disadvantages of using bone marrow as a source. Human trabecular bone-derived cell populations are pluripotent stem cells, which have multilineage potential and can give rise not only to osteoblasts, but also to adipocytes, when subjected to appropriate treatment protocols (1). Sottile et al (2) compared the characteristics of mesenchymal cell cultures established either from trabecular bone or from bone marrow, and the results showed that both of the cell cultures actually had similar characteristics to bone marrow-derived MSCs, differentiating into osteoblasts, chondrocytes and adipocytes under appropriate differentiating conditions. Therefore, trabecular bone tissue is a good source of adult MSCs for in vitro investigation.

The fate and commitment of MSCs are regulated by microenvironmental conditions, such as injury, inflammation and tumors. For example, under conditions of chronic inflammation, MSCs may contribute to adverse manifestations, such as the accumulation of fat deposits in bone and muscles, impaired healing and fibrosis after severe injury, or altered hematopoiesis and autoimmunity (3). On the other hand, MSCs also exert anti-inflammatory effects that are important in maintaining homeostatic balance. To date, MSCs have been used as a treatment modality for several inflammation-related diseases, such as inflammatory bowel disease (IBD) (4), graft vs. host disease (5), rheumatoid arthritis (6) and multiple sclerosis (7). In addition, MSCs secrete large amounts of inflammatory cytokines which regulate the inflammatory process. Thus, these findings indicate a complex, functional interaction between MSCs and the inflammatory microenvironment. 
T lymphocytes play a central role in the initiation and maintenance of inflammatory processes. Accumulation of $\mathrm{T}$ cells at inflammatory sites is one of the characteristic features of chronic inflammatory diseases (8). During the development of inflammation, $\mathrm{T}$ cells are activated by inflammatory messengers, and 'activated' $\mathrm{T}$ cells are a sign of ongoing inflammation. $T$ cell activation depends on signals delivered from antigen-presenting cells (APCs) through triggering of their $\mathrm{T}$ cell receptor (TCR) complex and a co-stimulatory receptor such as CD28 (9). The T cell activation pathway is triggered when a $\mathrm{T}$ cell encounters its cognate antigen, such as plant mitogen phytohemagglutinin (PHA), which has a marked selectivity for $\mathrm{T}$ lymphocytes. Following short-term pre-incubation with PHA, T cell activity is maintained. $\mathrm{T}$ cells activated with PHA express Ia-like antigens (which play a role in the stimulation of $\mathrm{T}$ lymphocytes by autologous PHA-T lymphocytes) and acquire the ability to stimulate autologous $\mathrm{T}$ lymphocytes in mixed lymphocyte reaction (10). Therefore, $\mathrm{T}$ cell activation using PHA stimulation is a good pathway with which to curb inflammation.

To better understand the effects of $\mathrm{T}$ cell activation and inflammation on the adipogenic and osteogenic differentiation of MSCs, PHA-activated T cells were used for co-culturing with MSCs in the present study. The inflammatory gene expression was detected by quantitative RT-PCR, and the adipo- and osteo-specific proteins were determined by western blotting. TGF- $\beta /$ Smad signaling was described by western blotting; and ALP activity was detected by pNPP method; and the adipo-/osteo-differentiation of MSCs was further verified using histological and immunohistochemical staining.

\section{Materials and methods}

All the procedures were approved by the Ethics Committee of the Union Hospital and the Tongji Medical College. Informed consent was obtained from the patients included in the study.

Isolation and culture of MSCs. Human trabecular bonederived MSCs were isolated from patients undergoing total hip replacement. One piece of bone was collected from the removed femoral neck under sterilization. The bone was rinsed several times (3-5 times) with PBS to remove excess blood. Subsequently, the bone was placed onto a sterile Petri dish and cancellous bone was broken into $2-3 \mathrm{~mm}^{2} \times 1 \mathrm{~mm}$ fragments (the cortical bone was discarded). Subsequently, the bone fragments were placed into $15-\mathrm{ml}$ centrifuge tubes in collagenase solution $(3 \mathrm{mg} / \mathrm{ml})$, and the tubes were incubated on a rotator at $37^{\circ} \mathrm{C}$ for $3 \mathrm{~h}$. After $3 \mathrm{~h}$ of digestion, the same volume of complete culture medium (CM) was added to neutralize the reaction, and the supernatant was filtered through a $70-\mu \mathrm{m}$ cell strainer. Finally, we obtained MSC solution, the cell density was adjusted and cells were seeded in T-75 cell culture flasks. The flask was placed in a humidified incubator at $37^{\circ} \mathrm{C}$ with $5 \%$ $\mathrm{CO}_{2}$. After a 24-h culture, the culture medium was refreshed; the culture medium was replaced every 2-3 days.

In vitro osteogenic, adipogenic and chondrogenic differentiation of MSCs. MSCs of passage 3-5 were used in the experiments. MSCs were plated in a 24-well plate in coverslips at a density of $1.5 \times 10^{4}$ cells $/ \mathrm{ml}$. For osteogenic differentiation, after becoming confluent, the cells were incubated in an osteogenic medium (OS) containing dexamethasone, ascorbate-phosphate and $\beta$-glycerolphosphate in complete medium. OS was replaced every 2-3 days for 3 weeks. The cells were fixed using 4\% paraformaldehyde, and subjected to Alizarin Red S staining and immunostaining (mouse anti-human osteocalcin monoclonal antibody, osteogenic marker). For adipogenic differentiation, MSCs were seeded at a density of $7.4 \times 10^{4}$ cells $/ \mathrm{ml}$. The subconfluent cells were cultured in adipogenic differentiation medium, containing hydrocortisone, isobutylmethylxanthine and indomethacin in complete medium. After 7-21 days of stimulation, the cells were fixed and then detected using Oil Red staining and immunostaining (goat anti-mouse FABP-4 polyclonal antibody, adipogenic marker). As previously described, a pellet culture system was used for chondrogenic differentiation. A total of $2.5 \times 10^{5}$ cells were transferred into a $15-\mathrm{ml}$ tube and pelleted by spin-down. The pellet was cultured in $0.5 \mathrm{ml}$ of chondrogenic differentiation medium, containing dexamethasone, ascorbate-phosphate, proline, pyruvate, TGF- $\beta 3$, insulin, transferrin and selenious acid in complete medium. The chondrogenic culture medium was changed every 2-3 days for 3 weeks. The pellets were then fixed and subjected to frozen sections. Immunostaining method was used to detect the expression of aggrecan protein (goat anti-human aggrecan polyclonal antibody, chondrogenic marker).

$T$ cell isolation and activation. Peripheral blood mononuclear cells (PBMCs) were collected from healthy donors and separated by Ficoll-Hypaque density gradient centrifugation. The $\mathrm{T}$ cells were then isolated and purified from PBMCs by magnetic-activated cell sorting using the CD3 MicroBead-based isolation kit according to the manufacturer's instructions (MACS; Miltenyi Biotec). To achieve higher purities, the positively selected $\mathrm{CD}^{+}$cell fractions were separated again over a new, freshly prepared LS column. The viability of $\mathrm{CD}^{+}$cell fractions was measured by trypan blue, with cells generally being $>95 \%$. For activation, $\mathrm{CD}^{+}$cell fractions ( $\mathrm{T}$ cells) were resuspended in $\alpha$-MEM medium containing PHA $(2.5 \mu \mathrm{g} / \mathrm{ml}), 10 \%$ FCS, penicillin $(100 \mathrm{U} / \mathrm{ml})$, streptomycin $(100 \mu \mathrm{g} / \mathrm{ml})$, and L-glutamine $(2 \mathrm{mM})$ and incubated at $37^{\circ} \mathrm{C}$ for 2 days in tissue culture tubes with filtered caps.

MTS assay for cell proliferation. MTS assay was used to detect the cell proliferation of MSCs after treatment with different concentrations of PHA-activated T cells. MSCs were placed into each well of 96-well plates at a density of $6 \times 10^{3}$ cells $/ \mathrm{ml}$. At $24 \mathrm{~h}$ after plating for attachment, cells were incubated with different concentrations of activated T cells (6x10 to $6 \times 10^{5}$ cells $\left./ \mathrm{ml}\right)$. On day 4 , suspended cells were removed by gentle washing with phosphate-buffered saline (PBS). The number of adherent cells remaining in each well was then quantified using a coupled enzymatic assay, which resulted in the conversion of a tetrazolium salt into a red formazan product (MTS assay). Recording of the absorbance at $490 \mathrm{~nm}$ in the MTS assay was carried out.

MSC differentiation under $T$ cell activation and inflammation. MSCs were co-cultured with activated $\mathrm{T}$ cells to mimic the inflammatory microenvironment. In the experiment, the cells were divided into the control group and inflammatory 
group (inflammatory). In the inflammatory group, MSCs were seeded onto plates at a concentration of $1.2 \times 10^{4}$ cells $/ \mathrm{ml}$ in $\mathrm{CM}$, containing 10\% FCS, $100 \mu \mathrm{g}$ PSN (penicillin, streptomycin and neomycin) and $100 \mu \mathrm{M} \mathrm{L}$-ascorbic acid phosphate in $\alpha$-MEM. Activated T cells were then added at a concentration of $0.6 \times 10^{5}$ cells $/ \mathrm{ml}$, after the cultures became $90 \%$ confluent. In the control groups, MSCs were cultured without $\mathrm{T}$ cell exposure. The cultures were refreshed with complete medium (CM) every 2-3 days for the following measurements.

$R N A$ isolation and quantitative RT-PCR. To investigate the effects of the inflammatory microenvironment induced by activated T cells, quantitative RT-PCR was used to detect the expression of inflammatory genes and osteo-/adipo-specific genes. After a 1-day co-culture with activated T cells, MSCs were collected in TRIzol reagent (Invitrogen, Carlsbad, CA, USA), followed by RNA isolation according to the manufacturer's instructions. The RNA samples were subjected to cDNA synthesis, followed by quantitative PCR assays. The reverse transcriptase reaction was carried out using ThermoScript ${ }^{\mathrm{TM}}$ reverse transcription reagents (Roche Applied Science). PCRs were performed according to the real-time PCR machine manufacturer's instructions (MJ Research, Inc., Watertown, MA, USA), which allow real-time quantitative detection of the PCR product by measuring the increase in SYBR-Green fluorescence caused by binding of SYBR-Green (Bio-Rad Laboratories, Québec, Canada) to double-stranded DNA. To amplify specific gene products, the following primers were used: IL-6 (forward, CCTCGACGGCATCTCAGCCC and reverse, TGCCCAGTGGACAGGTTTCTGAC); IL-10 (forward, CAAGGCCGTGGAGCAGGTGAA and reverse, GGTTTCTCAAGGGGCTGGGTCA); INF $\gamma$ (forward, TCG CCAGCAGCTAAAACAGGGA and reverse, GCTGCCTA GTTGGCCCCTGA); PPAR- $\gamma$ (forward, GCTGTTATGGGT GAAACTCT and reverse, ATGGAATGTCTTCGTAATGT); RUNX2 (forward, GTGCCTAGGCGCATTTCA and reverse: GCTCTTCTTACTGAGAGTGGAAGG); and 18S rRNA (forward, CCGCAGCTAGGAATAATGGAATA and reverse, TCTAGCGGCGCAATACGAAT), which served as an internal control. Amplification was performed using a profile at $94^{\circ} \mathrm{C}$ for $1 \mathrm{~min}$ (denaturation), $60^{\circ} \mathrm{C}$ for $30 \mathrm{sec}$ (annealing), $72^{\circ} \mathrm{C}$ for $45 \mathrm{sec}$ (elongation) for a total of 38 cycles, followed at the end by $72^{\circ} \mathrm{C}$ for $5 \mathrm{~min}$ (extension). Negative controls without RT were carried out in parallel for every PCR reaction to exclude amplification of contaminating DNA.

Western blot analysis. Under the inflammatory microenvironment induced by activated T cells, cell lysates of MSCs were collect at $0,1,3,6,12$ and $18 \mathrm{~h}$ and stored at $-20^{\circ} \mathrm{C}$ for western blot analysis. The protein concentrations were measured using the Bio-Rad protein assay, and equalized protein samples were used for the electrophoresis. The proteins were then electro-transferred to $0.45 \mu \mathrm{m}$-pore-diameter polyvinylidene difluoride (PVDF) membranes (Invitrogen). PVDF membranes were blocked in blocking agent (5\% skim milk, $0.1 \%$ Tween-20 in basic buffer) overnight at $4^{\circ} \mathrm{C}$. After blocking of non-specific immunoglobulin (IgG) binding, the membranes were incubated in primary antibodies at a 1:400 dilution for $2 \mathrm{~h}$ at $4^{\circ} \mathrm{C}$ under rocking. The membranes were then incubated for $2 \mathrm{~h}$ at $4^{\circ} \mathrm{C}$ with a 1:2,000 dilution of goat secondary anti- bodies in antibody diluents. Finally, the ECL-Plus western blotting system was used, and immunoreactive bands were revealed and quantified using ImageQuant LAS 400 software (GE Healthcare Life Sciences). The primary antibodies were anti-PPAR- $\gamma$, anti-RUNX2, anti-TGF- $\beta 1$, anti-Smad3 and anti-P-Smad3 produced in rabbit (Sigma-Aldrich).

Immunohistochemical staining. After 2-3 weeks of culture, the cells were fixed and saved for immunostaining, to assess the possible adipogenic or osteogenic differentiation of MSCs. The culture medium was aspirated and the cells were washed twice PBS. The cells were fixed with $4 \%$ paraformaldehyde for $20 \mathrm{~min}$ at room temperature. After fixation, the cultures were washed with $1 \%$ BSA in PBS and blocked with $0.3 \%$ Triton X-100, 1\% BSA, $10 \%$ normal donkey serum in PBS for $45 \mathrm{~min}$. Subsequently, the primary antibody was added and incubated overnight at $4^{\circ} \mathrm{C}$. After washing, the secondary antibody was added and incubation was carried out in the dark for $60 \mathrm{~min}$ at room temperature. The primary antibodies used in the experiment were goat anti-human FABP4 and mouse anti-human osteocalcin antibodies (R\&D Systems); and the secondary antibodies were fluorochrome-conjugated donkey anti-goat and donkey anti-mouse (R\&D Systems). Staining was examined using fluorescence microscopy immediately.

Oil Red $O$ staining and Alizarin Red staining. To determine whether the inflammatory microenvironment induced by activated T cells affects the differentiation of MSCs, the adipogenic and osteogenic differentiation of the cells was evaluated after 2-3 weeks of culture. Briefly, the cultures were washed with PBS, $2.5 \%$ glutaraldehyde was added $(0.5 \mathrm{ml} /$ well) (24-well plate), and the reaction was carried out at room temperature for $20 \mathrm{~min}$. For adipogenic differentiation, Oil Red O solution was added to the fixed cells; for osteogenic differentiation, $2 \%$ Alizarin Red solution was added. The plate was kept at $37^{\circ} \mathrm{C}$ in an incubator for 10-20 min. The staining was monitored under a microscope every 2-5 min and images were captured under the microscope immediately for analysis.

In vitro osteogenic differentiation and pNPP assay. For osteogenic differentiation, the cells were cultured in OS, containing dexamethasone, ascorbate-phosphate and $\beta$-glycerolphosphate in CM. After 2-3 weeks of culture, the cells were subjected to pNPP alkaline phosphatase assay to detect alkaline phosphatase (ALP) activity. Assay buffer $1 \mathrm{X}$ was prepared and the cells were gently washed twice with the buffer. Then, $300 \mu \mathrm{l}$ of $1 \mathrm{X}$ assay buffer in Triton X-100 per well (6-well plate) was added to the cells, and the adherent cells were scraped off and transferred into a microcentrifuge tube. The cell suspension was incubated at $4^{\circ} \mathrm{C}$ for 10 min under agitation. After centrifugation, the supernatant was collected for alkaline phosphatase assay, according to the instructions for the SensoLyte ${ }^{\circledR}$ pNPP alkaline phosphatase assay kit. Meanwhile, the concentrations of the proteins were determined using Bio-Rad protein assay. The results of ALP activity was determined using the standard curve and normalized by the total protein content. ALP activity $=\operatorname{ALP}(\mu \mathrm{g} / \mathrm{ml}) /$ protein $(\mathrm{mg} / \mathrm{ml})$.

Statistical analysis. All experiments were repeated three times, and the results are expressed as the means \pm SEM. Data 


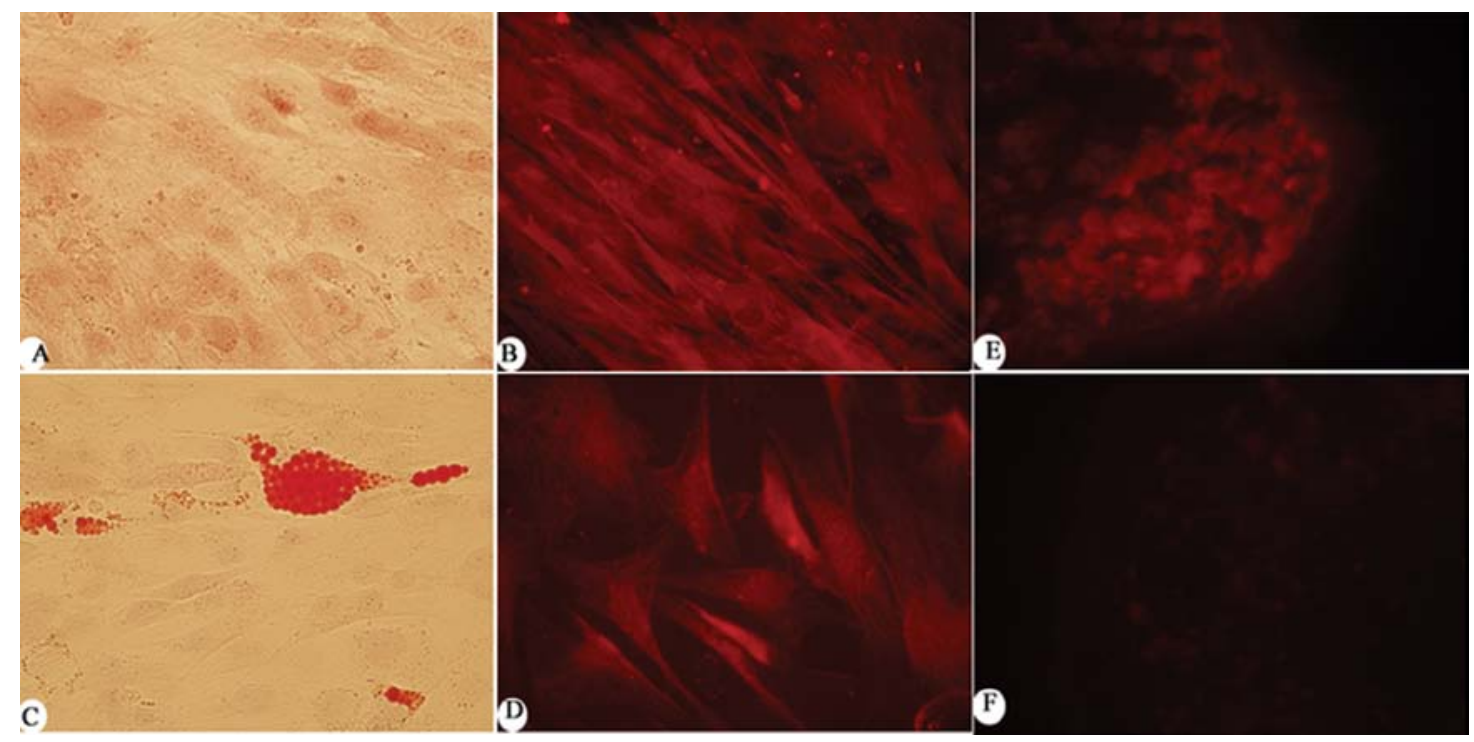

Figure 1. Characterization of MSCs isolated from human trabecular bone. In vitro osteogenic differentiation as detected by (A) Alizarin Red staining and (B) osteocalcin immunofluorescence. Adipogenic differentiation of MSC as determined by (C) Oil Red O staining and (D) FABP-4 immunofluorescence. (E) Chondrogenic differentiation as detected by aggrecan immunofluorescence. (F) Negative control (magnification, x60).

were analyzed by ANOVA or t-test, and $\mathrm{P}<0.05$ was considered to indicate a statistically significant result.

\section{Results}

Characterization of MSCs isolated from human trabecular bone. One of the defining characteristics of MSCs is their multilineage differentiation potential. Under certain inductive conditions, MSCs are able to acquire the characteristics of cells derived from the embryonic mesoderm, such as osteoblasts, chondrocytes and adipocytes. In order to define the characteristics of the trabecular bone-derived MSCs, cell type-specific histological and immunochemical staining were used. To identify adipogenic and osteogenic differentiation in vitro, the cultures were stained with Oil Red $\mathrm{O}$ and Alizarin Red S, respectively. Trabecular bone-derived MSC differentiation also was verified by analyzing the gene expression of adipogenic, chondrogenic and osteogenic markers, such as osteocalcin, FABP-4 and aggrecan (Fig. 1). The results showed that the MSCs were successfully differentiated into adipocytes, osteocytes and chondrocytes.

MSC proliferation with PHA-activated T cell supplements. MTS is a common and useful method for monitoring cell proliferation, for the detection of viable cells. To quantify the proliferation of trabecular bone-derived MSCs following exposure to activated $\mathrm{T}$ cells of different concentrations, an MTS assay was performed (Fig. 2). The results showed that lower concentrations (ratios of 1:100 and 1:10) of T cells had no effect on the proliferation of MSCs. However, high concentrations of $\mathrm{T}$ cells significantly increased the proliferation of MSCs, particularly at ratios of 10:1 and 100:1 ( $\mathrm{P}<0.01)$.

Expression of inflammatory factors induced by T-cell exposure. $\mathrm{T}$ cells induce the expression of genes mostly related to inflammatory cytokines, which are thought to play an important role in the process of chronic inflammation. The

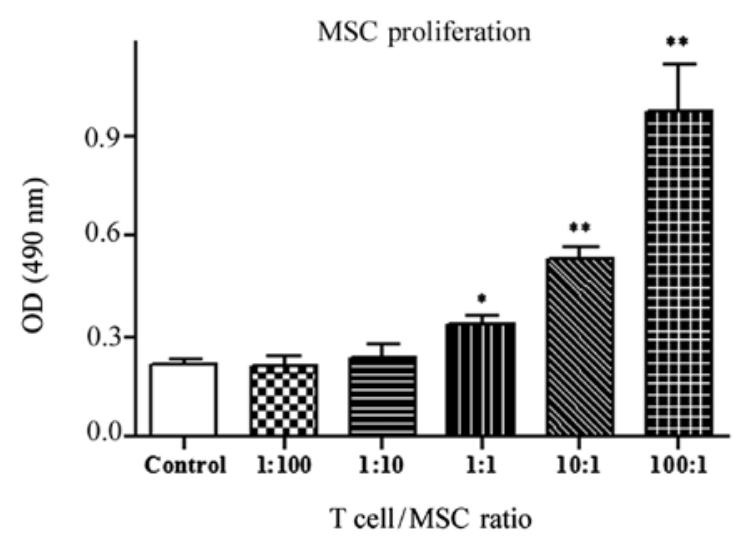

Figure 2. Effect of activated $\mathrm{T}$ cells on the proliferation of trabecular bonederived MSCs as assessed by the MTS test. Data represent the means \pm SEM. Each experiment was conducted in triplicate. ${ }^{*} \mathrm{P}<0.05,{ }^{* *} \mathrm{P}<0.01$.

present study was undertaken to detect the gene expression profile of MSCs exposed to activated T cells using qRT-PCR. The results showed that the expression of the proinflammatory gene IL-6 was significantly upregulated (6.89-fold compared with the control) and acted to promote excessive inflammation. On the other hand, the expression of anti-inflammatory genes, such as IL-10 (-44.24-fold) and INF (-5.96-fold), was significantly downregulated when compared to the control group (Fig. 3). Therefore, T-cell exposure increased the expression of proinflammatory genes but inhibited the expression of anti-inflammatory genes and led to an excessive inflammatory microenvironment.

Adipo-/osteo-specific gene expression is influenced by activated $T$ cells and inflammation. In order to investigate the effects of an inflammatory microenvironment induced by activated $\mathrm{T}$ cells on the adipogenic and osteogenic differentiation of MSCs, qRT-PCR and western blotting were performed. The 
A

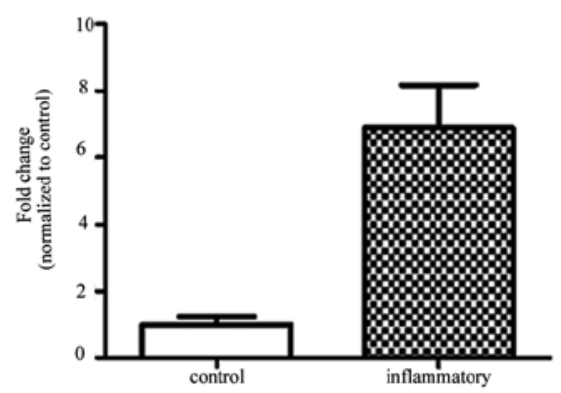

B

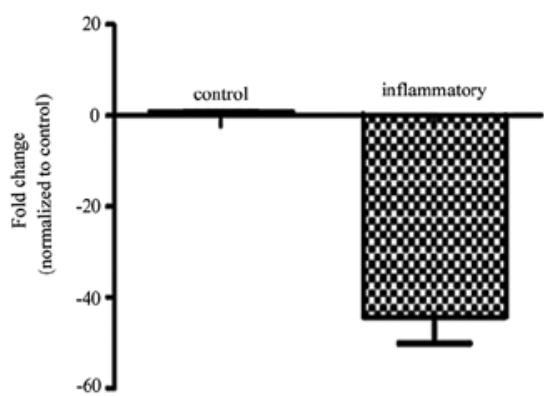

C

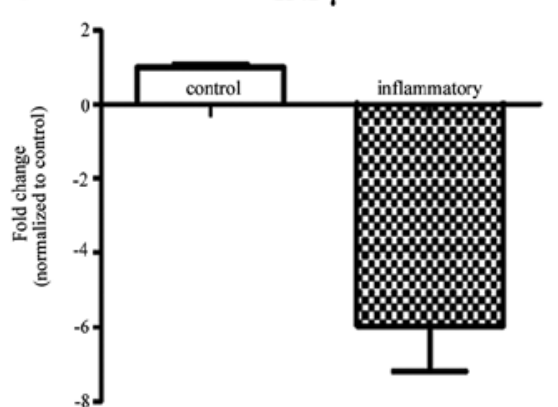

Figure 3. Expression of genes in MSCs following exposure to activated T cells. qRT-PCR was used to detect the expression of pro-inflammatory gene (IL-6) and anti-inflammatory genes (IL-10, INF $\gamma$ ). mRNA expression of (A) IL-6, (B) IL-10 and (C) INF $\gamma$. Results are presented as the means \pm SEM of qPCR reaction triplicates prepared from the same sample.

$\mathbf{A}$

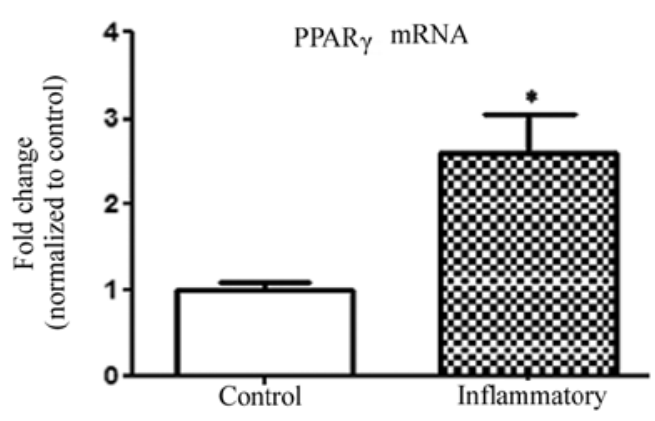

C

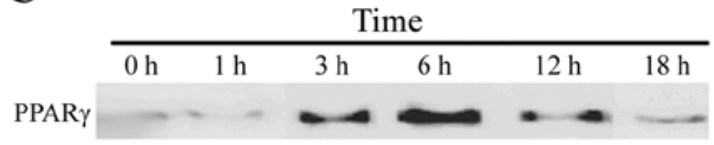

RUNX2

Actin
B

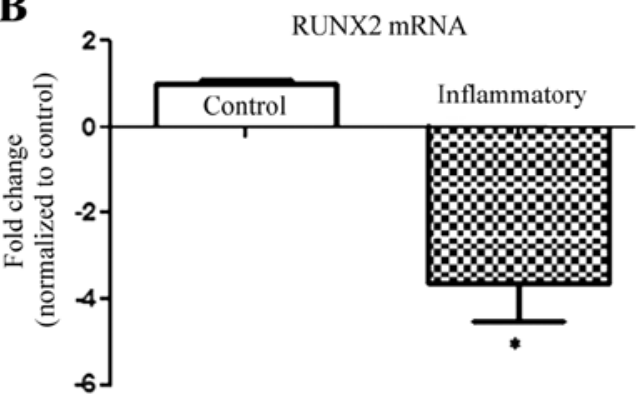

D

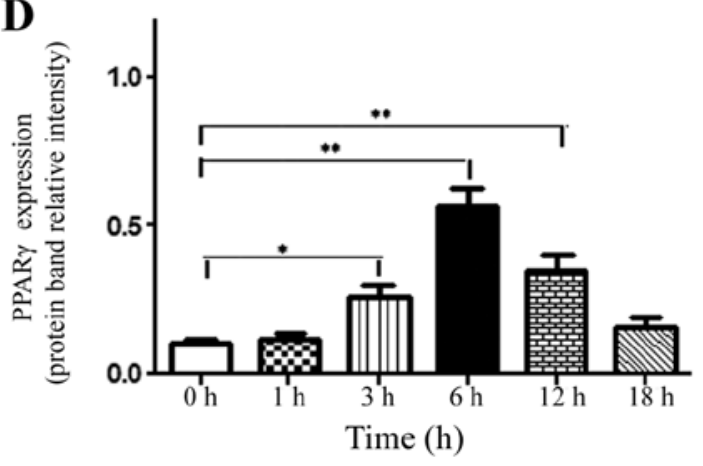

Figure 4. Effect of activated T cells and inflammation on the expression of adipogenic and osteogenic genes. qRT-PCR was used to detect the mRNA expression of (A) PPAR $\gamma$ and (B) RUNX2. Western blot analysis of cell lysates from the different groups at different time-points was conducted to determine the expression of PPAR $\gamma$ and RUNX2. Actin was used as a loading control. (C) PPAR $\gamma$ and RUNX2 protein expression; (D) PPAR $\gamma$ protein levels relative to actin protein level were assessed by densitometric analysis. Each value is the mean \pm SEM. ${ }^{*} \mathrm{P}<.05 ;{ }^{* *} \mathrm{P}<.001$.

results of qRT-PCR showed that the adipo-specific gene expression of PPAR $\gamma$ was significantly upregulated $(2.58$-fold compared with the control); while the osteogenic-specific gene expression of RUNX2 was significantly downregulated (-3.63-fold) (Fig. 4A and B). Western blot analysis revealed that the expression level of PPAR $\gamma$ was upregulated in MSCs after inflammatory stimulation with activated $\mathrm{T}$ cells, reaching a peak value at $6 \mathrm{~h}$, which was subsequently decreased (Fig. 4C). In contrast, MSCs expressed barely detectable levels of RUNX2 protein for the duration of the experiment; thus, we could not compare the expression levels of RUNX2 protein between the groups. However, the results still indicated that the alterations resulted in increased adipogenic differentiation and decreased osteogenic differentiation of MSCs at an early stage.
Adipogenic differentiation of MSCs is regulated by activated $T$ cells. MSCs were cultured in CM supplied with activated $\mathrm{T}$ cells. After a 2 -week culture, the cultures were fixed; half of them were subjected to Oil Red O staining, and the other half were used for the detection of the expression of FABP4 using fluorescence immunohistochemistry. The fluorescence staining showed a very weak diffuse fluorescence pattern in the control group (Fig. 5A) but strong FABP4 expression of MSCs in the inflammatory group (Fig. 5B). The results of Oil Red O staining showed accumulated intracellular lipid droplets in the inflammatory group (Fig. 5D), while no lipid droplet was found in the control group (Fig. 5C). Both types of staining indicated that an inflammatory microenvironment induced by activated $\mathrm{T}$ cells promoted the adipogenic differentiation of MSCs. 


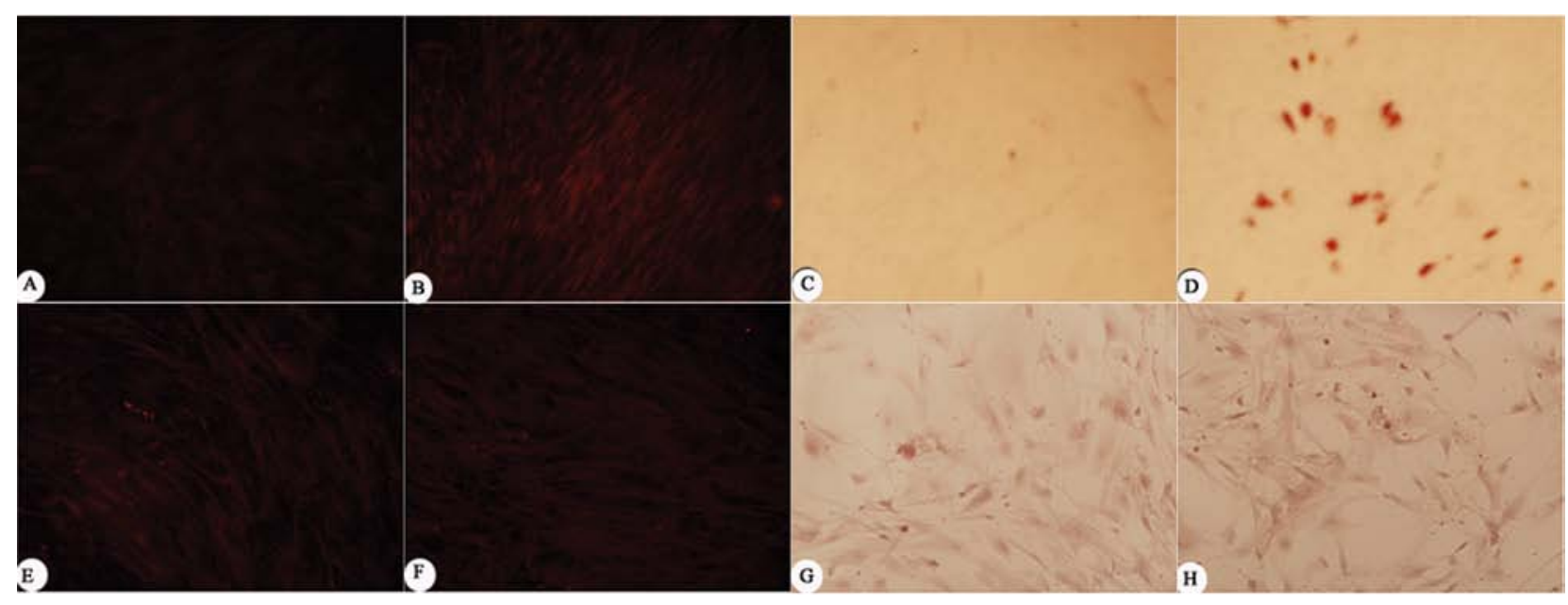

Figure 5. Effect of the T-cell-mediated inflammatory microenvironment on the adipogenic/osteogenic differentiation of MSCs. Fluorescent anti-FABP4 staining in (A) the control group and (B) inflammatory group. Oil Red O staining in (C) the control group and (D) inflammatory group. Fluorescent anti-osteocalcin staining in (E) the control group and (F) inflammatory group. Alizarin Red staining in (G) the control group and (H) inflammatory group.

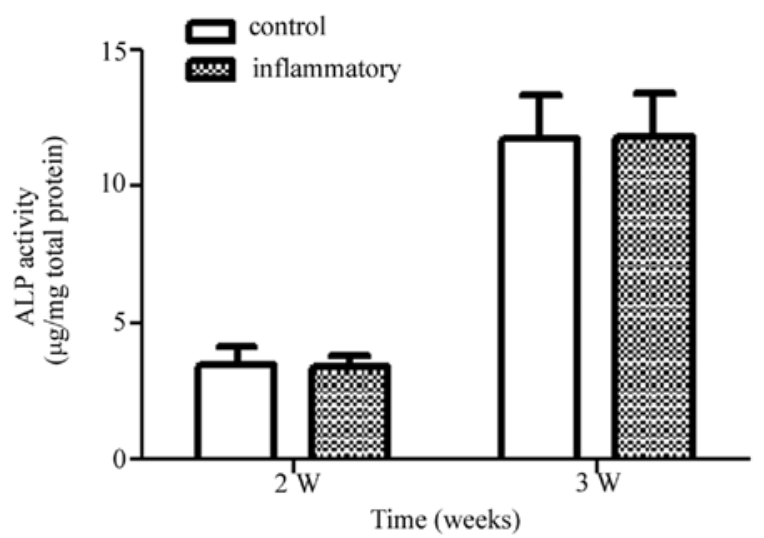

Figure 6. pNPP assay for the detection of ALP activity. The statistical diagram showed that the inflammatory microenvironment induced by activated $\mathrm{T}$ cells had no significant effect on ALP activity, as compared to the control group. ALP activity $(\mu \mathrm{g} / \mathrm{mg})$ in the different groups was normalized to the total protein. Results are presented as the means \pm SEM.

Osteogenic differentiation of MSCs in the presence of activated $T$ cells. In order to investigate the effect of activated $\mathrm{T}$ cells and inflammation on the osteogenic differentiation of MSCs, fluorescence immunohistochemical staining and Alizarin Red staining were used. The fluorescence staining showed that MSCs expressed lower levels of osteocalcin in both the inflammatory group and the control group. Alizarin Red staining showed similar results; only mild mineralization was observed in both of the groups (Fig. 5E-H). These results confirmed the finding that the inflammatory microenvironment induced by activated $\mathrm{T}$ cells had no significant effect on the osteogenic differentiation of MSCs.

ALP activity as determined by $p N P P$ method. The activity of ALP was detected to evaluate the effect of activated T cells on the osteogenic differentiation of MSCs in OS medium by pNPP method. The ALP levels expressed by MSCs increased with time in both the control and inflammatory groups in the presence of OS medium. However, at the time-points studied (week 2 and 3), no significant difference was noted in ALP activity due to the inflammatory microenvironment induced by the activated $\mathrm{T}$ cells, when compared to the control group (Fig. 6). Therefore, the results confirmed that the activated T cells had no obvious effects on the osteogenic differentiation of MSCs at the late stage.

TGF- $\beta /$ Smad3 signaling. To evaluate the effect of $\mathrm{T}$ cell treatment on the TGF- $\beta$ /smad pathway, the TGF- $\beta 1$ level was detected by western blotting. The expression of the TGF- $\beta 1$ gene in MSCs decreased significantly after inflammatory stimulation with activated $\mathrm{T}$ cells at $6 \mathrm{~h}$, compared to the control group. In addition, the gene expression of Smad3 and the phosphorylation levels of the MSC culture in the absence or presence of $\mathrm{T}$ cells were investigated by western blotting. At this time-point, the phosphorylation level of Smad3 protein declined in the presence of the T cells, when compared to control group. Therefore, $\mathrm{T}$ cell treatment inhibited the expression of TGF- $\beta 1$, resulting in the weakening of the TGF- $\beta /$ Smad3 pathway and enhancing the adipogenic differentiation of MSCs (Fig. 7).

\section{Discussion}

Mesenchymal stem cells (MSCs) have chemotactic ability and appear to migrate to sites of inflammation (11), where they appear to play an active role in tissue remodeling. MSCs are generally isolated from an aspirate of bone marrow; in addition, MSCs have been isolated from bone marrow, periosteum, trabecular bone, adipose tissue, synovium, skeletal muscle and deciduous teeth (12). Trabecular bone-derived mesenchymal stem cells (MSCs) are multipotent cells, which can differentiate into a number of different types of cells, including osteocytes, adipocytes, chondrocytes, myocytes and neurocytes $(1,2)$. Differentiation of MSCs into different lineages of cells is strictly regulated by various instructive signals, and alteration or malfunction of this regulation results in pathological consequences, such as osteoporosis or a high bone mass phenotype $(13,14)$. In the present study, histological 
A

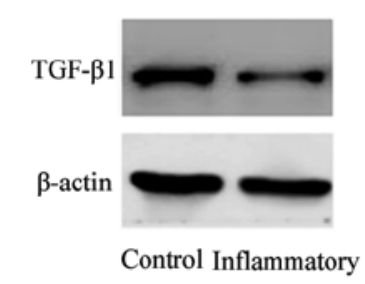

B

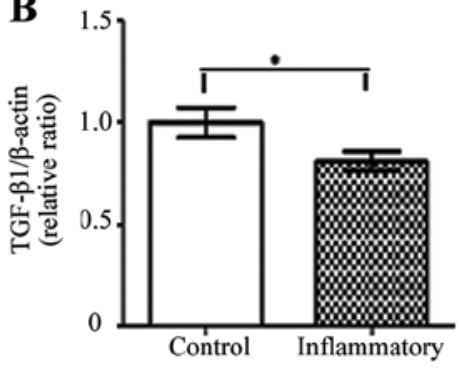

C

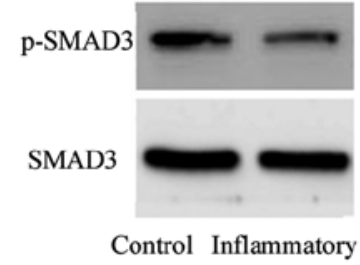

D

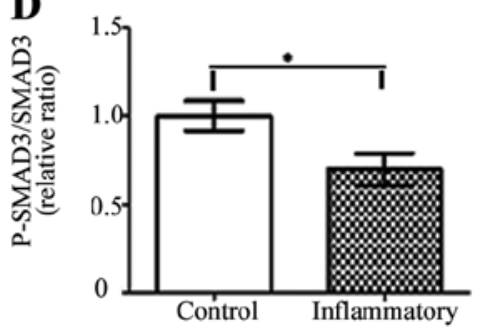

$\mathbf{E}$
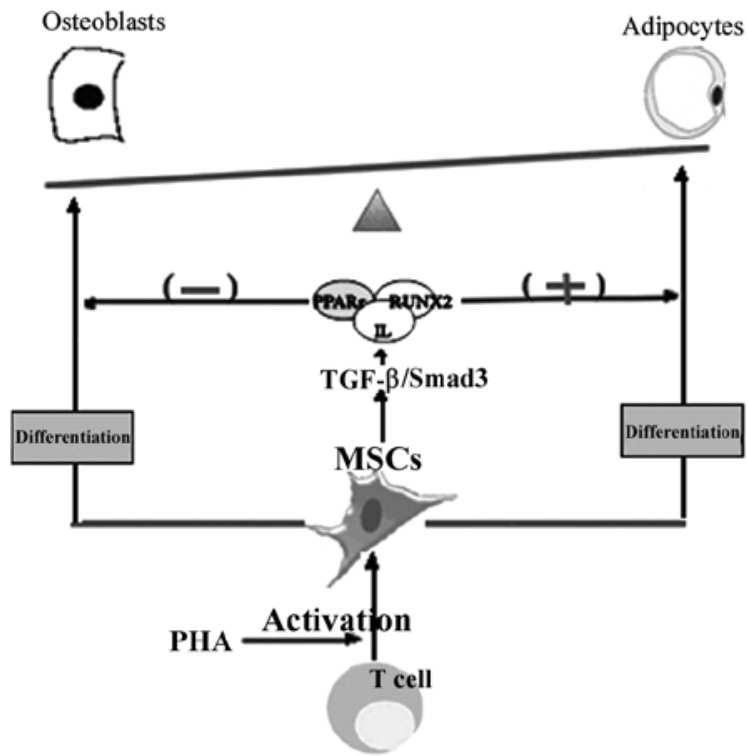

Figure 7. TGF- $\beta / \mathrm{Smad} 3$ signaling in the differentiation of MSCs treated with/without T cells. Quantification of multiple independent western blot assays showed the relative changes in TGF- $\beta 1$ (A) and phosphorylated levels of Smad3 (C) in MSC cultures. (B) TGF- $\beta 1$ protein levels relative to actin protein level were assessed by densitometric analysis. (D) Phosphorylation levels of Smad3 were assessed by densitometric analysis (data expressed as means \pm SD, $\mathrm{n}=6,{ }^{*} \mathrm{P}<0.05$, compared to the control group). (E) Proposed working model for activated $\mathrm{T}$ cells in the adipogenic/osteogenic differentiation of trabecular bone-derived MSCs.

and immunohistochemistry methods were used to confirm the multipotential characteristics of the trabecular bone-derived cells. Oil Red O staining and anti-FABP-4 staining showed trabecular bone-derived MSCs had adipogenic differentiation potential; Alizarin Red S staining and anti-osteocalcin staining showed osteogenic differentiation capability; and chondrogenesis of MSCs was evidenced by anti-aggrecan fluorescent staining. Therefore, the results showed that MSCs derived from trabecular bone were multipotent stem cells; that is, they can give rise to diverse cell types.

Another property of MSCs is the ability to reduce the proliferation of lymphocytes of various types $(15,16)$. For example, BM-MSCs have been reported to impair the proliferation of activated $\mathrm{T}$ cells (17). In contrast, activated $\mathrm{T}$ cells do affect the proliferation of in vitro cultured MSCs, but this depends on the concentration of the cells. In the present study, we examined the effects of various concentrations of activated T cells on the proliferation of MSCs by MST assay. The results showed that activated $\mathrm{T}$ cells had no effect on the proliferation of MSCs at low concentrations, but higher concentrations of activated $\mathrm{T}$ cells significantly increased the proliferation of MSCs, particularly at the ratios of 10:1 and 100:1 ( T cells/MSCs; $\mathrm{P}<0.01$ ). Therefore, in order to exclude effects of an increase in the proliferation of precursors on the differential outcome of MSCs; in subsequent experiments, $\mathrm{T}$ cells at a lower concentration were added to the cultures after MSCs reached $90 \%$ confluency.

Furthermore, one of the undisputed features of MSCs is their ability to produce a wide variety of chemokines and cytokine receptors, including those for tumor necrosis factor $\alpha$ (TNF- $\alpha$ ), interleukin (IL)-10, IL-6 and interferon $\gamma$ (INF $\gamma)$. The cytokine IL-10, which is produced by various immune cells, in particular monocytes/macrophages and
$\mathrm{T}$ cell subsets, has a crucial role in limiting the inflammatory response caused by activated T cells (18). MSCs are also known to produce high levels of IL-10 (19). What is more, MSCs constitutively expressed mRNA of IL-6, which acts as a predominantly pro-inflammatory cytokine. IL6 is produced by undifferentiated dividing MSCs, and the secretory pathway of MSCs is mediated through the activation of p38 MAPK (20). In addition, excessive release of INF $\gamma$ is associated with the pathogenesis of chronic inflammatory and autoimmune diseases. Previous research showed that INF $\gamma$ possesses both pro-inflammatory and anti-inflammatory effects depending on the pathophysiological conditions. INF $\gamma$ is pro-inflammatory in mouse models of diabetes, thyroiditis and myasthenia gravis, but anti-inflammatory in models such as experimental autoimmune encephalomyelitis, uveitis and collagen-induced arthritis (21).

Both MSCs and T cells express inflammatory cytokines, thus ELISA methods cannot be used directly to measure the expression levels of cytokines secreted by MSCs. Alternatively, the detection of mRNA expression was used. The expression of inflammatory genes was regulated in MSCs exposed to PHA-activated T cells. According to the results, IL-6 (pro-inflammatory gene) was significantly upregulated, while IL-10 and INF $\gamma$ (anti-inflammatory genes) were significantly downregulated. This indicated that the inflammatory microenvironment induced by activated $\mathrm{T}$ cells could increase the expression of proinflammatory genes but inhibited the expression of anti-inflammatory genes, leading to an excessive inflammatory microenvironment. Actually, these inflammatory factors not only attribute to inflammatory response but also play an important role in the differentiation of MSCs. Previous studies have shown that MSCs cultured in OS differentiated along the osteogenic lineage and downregulated 
protein and mRNA levels of IL-6 $(22,23)$. Our results are consistent with the findings of the adipogenic differentiation of MSCs following upregulated mRNA levels of IL-6.

The balance between peroxisome proliferator-activated receptor- $\gamma(\operatorname{PPAR} \gamma)$ and Runt-related transcription factor 2 (RUNX2) leads to MSC differentiating into adipocytes or osteoblasts, respectively. PPAR $\gamma$ is a member of the nuclear receptor superfamily of ligand-activated transcriptional factors, which act as a key regulator of adipogenesis. PPAR $\gamma$ is commonly termed the master regulator of adipogenesis; no factor has yet been identified that can induce normal adipogenesis in the absence of PPAR $\gamma$ (24). In contrast, RUNX2 promotes osteogenesis but inhibits adipogenesis of MSCs. These master regulators of different lineages are expressed at low levels in undifferentiated cells, maintaining the differentiation potential of MSCs $(25,26)$. In the experiment, expression of PPAR $\gamma$ and RUNX2 genes was detected by qRT-PCR and western blot analyses. The results showed that PHA-activated $\mathrm{T}$ cells significantly upregulated the expression of PPAR $\gamma$ (adipo-specific gene) in MSCs at both the RNA and protein levels. However, the expression of RUNX2 was altered at the RNA level but not at the protein level. qRT-PCR showed that the expression of RUNX2 was significantly downregulated in MSCs treated with activated T cells. Whether treated or not with activated $\mathrm{T}$ cells and inflammation, RUNX2 protein expressed by MSCs remained at or below the limits of detection for the duration of the experiment. Thus, it was difficult to compare the expression levels between the groups. According to the results, activated $\mathrm{T}$ cells and inflammation promoted adipogenesis in MSCs and it may play a role in the early stage of MSC osteogenesis.

To carry out the specialized functions of $\mathrm{T}$ cell activation and inflammation, the adipogenic differentiation of MSCs was further verified using histological and immunohistochemical staining. Fatty acid binding protein 4 (FABP4), also called aP2 (adipocyte protein 2), has a high affinity for a variety of fatty acids and facilitates their storage, trafficking and solubilization (27). FABP4 has been used as a marker (specific gene) to follow the differentiation of adipocytes. The expression of FABP4 can be induced by fatty acids, likely through changes in PPAR $\gamma$ expression or activity, both at the transcriptional and post-transcriptional level $(28,29)$. In accordance with its stimulatory effect on PPAR $\gamma$ expression, we found that PHA-activated T cells upregulated the expression of FABP4 in MSCs, as detected by fluorescence immunohistochemistry. In addition, the increase in FABP4 expression by activated $\mathrm{T}$ cells was consistent with the observed increase in Oil Red $\mathrm{O}$ staining. Therefore, these studies confirmed once again that PHA-activated T cells promoted MSC adipogenesis, by increasing the expression of key adipogenic genes.

Whether or not $\mathrm{T}$ cell activation and inflammation have an effect at later stages of osteogenic differentiation from MSCs was investigated. The osteogenic differentiation of MSCs was evaluated by the expression of osteocalcin, mineralization and alkaline phosphatase (ALP) activity. According to the results, it had no obvious effect on osteogenic differentiation from MSCs. Osteocalcin (bone gla-protein), secreted by osteoblasts, is generally regarded as an osteoblast-specific marker (30). The cells showed low-level expression of osteocalcin and mild mineralization in both groups, as evidenced by Alizarin Red S staining and fluorescence immunohistochemical staining. The findings were consistent with the protein expression of RUNX2. No significance was observed between the groups. Alkaline phosphatase (ALP) activity is another marker for osteoblast differentiation (31). Under osteogenic conditions (OS), pNPP assay revealed that activated T cells had no significant effect on ALP activity on the 14th and 21st day of osteogenic differentiation. Totally, these results indicated that PHA-activated $\mathrm{T}$ cells promote adipogenesis without affecting the osteogenesis of MSCs.

Growth factors such as transforming growth factor- $\beta 1$ (TGF- $\beta 1$ ) are known to inhibit adipocyte differentiation in vitro. MSCs can produce significant levels of transforming growth factor- $\beta 1$ (TGF- $\beta 1$ ) which can be further enhanced by anti-inflammatory cytokines (32). Moreover, Smad proteins play a key role in the regulation of TGF- $\beta$ signaling and Smad3 is the central intracellular mediator of TGF $\beta$ signaling. After activation of Smads, the effectors of TGF $\beta$ signaling result in Smad translocation from the cytoplasm into the nucleus where they act as transcriptional comodulators to regulate target gene expression (33). The TGF- $\beta / \mathrm{Smad} 3$ signaling pathway was found to play a key role in adipogenesis, which inhibited adipogenesis independent from the Wnt and $\beta$-catenin pathway (34). TGF- $\beta$ inhibits adipocyte differentiation. Previous results indicate that endogenous TGF- $\beta$ signaling regulates the rate of adipogenesis, and that $\mathrm{Smad} 3$ has distinct functions in this endogenous control of differentiation (35). TGF- $\beta$ targets the transcription factor cascade upstream of PPAR $\gamma$, and the inhibition of adipogenesis by TGF- $\beta$ was accompanied by reduced mRNA and protein levels of PPAR $\gamma$. In the present study, western blot analysis confirmed that $\mathrm{T}$ cell treatment inhibited the protein expression of TGF- $\beta 1$ and the phosphorylation of Smad3, resulting in a weakening of the TGF- $\beta /$ Smad pathway which enhanced the adipogenic differentiation of MSCs (Fig. 7).

In conclusion, we demonstrated the effects of $\mathrm{T}$ cell activation and inflammation on osteoblast and adipocyte differentiation of MSCs. The results showed that PHA-activated $\mathrm{T}$ cells upregulated the expression of adipocyte-specific genes and led to adipogenic differentiation, possibly due to gene expression of PPAR $\gamma$ which plays a more decisive role in the differentiation of MSCs exposed to activated T cells through TGF- $\beta /$ Smad 3 signaling. This may explain why we observed no obvious effect of PHA-activated T cells on osteogenic differentiation of MSCs, but it is complicated. Additionally, there are some limitations to the current research that merit further study. First, T cells, even activated $\mathrm{T}$ cells, normally have a short lifespan, which may have partially influenced the experimental outcomes. Thus, in future experiments, a constant supply of T cells will be necessary to observe the effects on MSC differentiation. Second, the present study mainly focused on the downstream regulatory events, and the precise molecular mechanisms and signaling pathways are still not clear. Thus, further study is needed to investigate the upstream regulatory mechanisms, particularly using knockdown techniques to elucidate the mechanism involved in the differential gene expression. In spite of these limitations of the present study, further investigation of the individual effects of activated T cells and inflammation on MSC differentiation in vivo is warranted. 


\section{Acknowledgements}

The authors appreciate the help of the Osteonecrosis Research Team of Union Hospital, Tongji Medical College, Wuhan, China. The present study was supported by the National Natural Science Foundation of China (no. 81201393).

\section{References}

1. Nuttall ME, Patton AJ, Olivera DL, Nadeau DP and Gowen M: Human trabecular bone cells are able to express both osteoblastic and adipocytic phenotype: implications for osteopenic disorders J Bone Miner Res 13: 371-382, 1998.

2. Sottile V, Halleux C, Bassilana F, Keller H and Seuwen K: Stem cell characteristics of human trabecular bone-derived cells. Bone 30: 699-704, 2002

3. Lepperdinger G: Inflammation and mesenchymal stem cell aging. Curr Opin Immunol 23: 518-524, 2011.

4. Garcia-Olmo D, Garcia-Arranz M, Garcia LG, Cuellar ES, Blanco IF, Prianes LA, Montes JA, Pinto FL, Marcos DH and Garcia-Sancho L: Autologous stem cell transplantation for treatment of rectovaginal fistula in perianal Crohn's disease: a new cell-based therapy. Int J Colorectal Dis 18: 451-454, 2003.

5. Le Blanc K, Frassoni F, Ball L, Locatelli F, Roelofs H, Lewis I, Lanino E, Sundberg B, Bernardo ME, Remberger M, Dini G, Egeler RM, Bacigalupo A, Fibbe W and Ringden O: Mesenchymal stem cells for treatment of steroid-resistant, severe, acute graft-versus-host disease: a phase II study. Lancet 371: 1579-1586, 2008.

6. Gonzalez-Rey E, Gonzalez MA, Varela N, O'Valle F, Hernandez-Cortes P, Rico L, Buscher D and Delgado M: Human adipose-derived mesenchymal stem cells reduce inflammatory and $\mathrm{T}$ cell responses and induce regulatory $\mathrm{T}$ cells in vitro in rheumatoid arthritis. Ann Rheum Dis 69: 241-248, 2010.

7. Gerdoni E, Gallo B, Casazza S, Musio S, Bonanni I, Pedemonte E, Mantegazza R, Frassoni F, Mancardi G, Pedotti R and Uccelli A: Mesenchymal stem cells effectively modulate pathogenic immune response in experimental autoimmune encephalomyelitis. Ann Neurol 61: 219-227, 2007.

8. Tabassam FH, Umehara H and Domae N: Beta 2-integrin, LFA-1-mediated p125FAK activation. J Osaka Dent Univ 33: 43-51, 1999.

9. Schmitz ML, Bacher S and Dienz O: NF- $\kappa$ B activation pathways induced by T cell costimulation. FASEB J 17: 2187-2193, 2003.

10. Russo C, Indiveri F, Quaranta V, Molinaro GA, Pellegrino MA and Ferrone S: Stimulation of human T lymphocytes by PHA-activated autologous T lymphocytes: analysis of the role of Ia-like antigens with monoclonal antibodies. Immunogenetics 12: 267-274, 1981.

11. Wang L, Li Y, Chen X, Chen J, Gautam SC, Xu Y and Chopp M: MCP-1, MIP-1, IL-8 and ischemic cerebral tissue enhance human bone marrow stromal cell migration in interface culture. Hematology 7: 113-117, 2002.

12. Barry FP and Murphy JM: Mesenchymal stem cells: clinical applications and biological characterization. Int J Biochem Cell Biol 36: 568-584, 2004

13. Duque G: Bone and fat connection in aging bone. Curr Opin Rheumatol 20: 429-434, 2008.

14. Huang J, Zhao L, Xing L and Chen D: MicroRNA-204 regulates Runx 2 protein expression and mesenchymal progenitor cell differentiation. Stem Cells 28: 357-364, 2010.

15. Le Blanc K: Immunomodulatory effects of fetal and adult mesenchymal stem cells. Cytotherapy 5: 485-489, 2003.

16. Krampera M, Glennie S, Dyson J, Scott D, Laylor R, Simpson E and Dazzi F: Bone marrow mesenchymal stem cells inhibit the response of naive and memory antigen-specific $\mathrm{T}$ cells to their cognate peptide. Blood 101: 3722-3729, 2003.

17. Bocelli-Tyndall C, Bracci L, Spagnoli G, Braccini A, Bouchenaki M, Ceredig R, Pistoia V, Martin I and Tyndall A: Bone marrow mesenchymal stromal cells (BM-MSCs) from healthy donors and auto-immune disease patients reduce the proliferation of autologous- and allogeneic-stimulated lymphocytes in vitro. Rheumatology 46: 403-408, 2007.
18. Scapini P, Lamagna C, Hu Y, Lee K, Tang Q, DeFranco AL and Lowell CA: B cell-derived IL-10 suppresses inflammatory disease in Lyn-deficient mice. Proc Natl Acad Sci USA 108: E823-E832, 2011.

19. Oh JY, Kim MK, Shin MS, Lee HJ, Ko JH, Wee WR and Lee JH: The anti-inflammatory and anti-angiogenic role of mesenchymal stem cells in corneal wound healing following chemical injury. Stem Cells 26: 1047-1055, 2008.

20. Yew TL, Hung YT, Li HY, Chen HW, Chen LL, Tsai KS, Chiou SH, Chao KC, Huang TF, Chen HL and Hung SC: Enhancement of wound healing by human multipotent stromal cell conditioned medium: the paracrine factors and p38 MAPK activation. Cell Transplant 20: 693-706, 2011.

21. Kelchtermans H,Billiau A and Matthys P: How interferon-gamma keeps autoimmune diseases in check. Trends Immunol 29: 479-486, 2008.

22. Majumdar MK, Thiede MA, Haynesworth SE, Bruder SP and Gerson SL: Human marrow-derived mesenchymal stem cells (MSCs) express hematopoietic cytokines and support long-term hematopoiesis when differentiated toward stromal and osteogenic lineages. J Hematother Stem Cell Res 9: 841-848, 2000.

23. Pricola KL, Kuhn NZ, Haleem-Smith H, Song Y and Tuan RS: Interleukin-6 maintains bone marrow-derived mesenchymal stem cell stemness by an ERK1/2-dependent mechanism. J Cell Biochem 108: 577-588, 2009.

24. Kawai $M$ and Rosen CJ: PPAR $\gamma$ : a circadian transcription factor in adipogenesis and osteogenesis. Nat Rev Endocrinol 6: 629-636, 2010.

25. Bennett CN, Longo KA, Wright WS, Suva LJ, Lane TF, Hankenson KD and MacDougald OA: Regulation of osteoblastogenesis and bone mass by Wnt10b. Proc Natl Acad Sci USA 102: 3324-3329, 2005.

26. Rosen ED and MacDougald OA: Adipocyte differentiation from the inside out. Nat Rev Mol Cell Biol 7: 885-896, 2006.

27. Reese-Wagoner A, Thompson J and Banaszak L: Structural properties of the adipocyte lipid binding protein. Biochim Biophys Acta 1441: 106-116, 1999.

28. Distel RJ, Robinson GS and Spiegelman BM: Fatty acid regulation of gene expression. Transcriptional and post-transcriptional mechanisms. J Biol Chem 267: 5937-5941, 1992.

29. Platt ID and El-Sohemy A: Regulation of osteoblast and adipocyte differentiation from human mesenchymal stem cells by conjugated linoleic acid. J Nutr Biochem 20: 956-964, 2009.

30. Kitamura S, Ohgushi H, Hirose M, Funaoka H, Takakura Y and Ito $\mathrm{H}$ : Osteogenic differentiation of human bone marrow-derived mesenchymal cells cultured on alumina ceramics. Artif Organs 28: 72-82, 2004.

31. van Dinther M, Visser N, de Gorter DJ, Doorn J, Goumans MJ, de Boer J and ten Dijke P: ALK2 R206H mutation linked to fibrodysplasia ossificans progressiva confers constitutive activity to the BMP type I receptor and sensitizes mesenchymal cells to BMP-induced osteoblast differentiation and bone formation. J Bone Miner Res 25: 1208-1215, 2010.

32. Tan X, Weng T, Zhang J, Wang J, Li W, Wan H, Lan Y, Cheng X, Hou N, Liu H, Ding J, Lin F, Yang R, Gao X, Chen D and Yang X: Smad4 is required for maintaining normal murine postnatal bone homeostasis. J Cell Sci 120: 2162-2170, 2007.

33. Roelen BA and Dijke P: Controlling mesenchymal stem cell differentiation by TGF $\beta$ family members. J Orthop Sci 8: 740-748, 2003.

34. Tsurutani Y, Fujimoto M, Takemoto M, Irisuna H, Koshizaka M, Onishi S, Ishikawa T, Mezawa M, He P, Honjo S, Maezawa Y, Saito $\mathrm{Y}$ and Yokote K: The roles of transforming growth factor- $\beta$ and Smad3 signaling in adipocyte differentiation and obesity. Biochem Biophys Res Commun 407: 68-73, 2011.

35. Choy L, Skillington J and Derynck R: Roles of autocrine TGF- $\beta$ receptor and Smad signaling in adipocyte differentiation. J Cell Biol 149: 667-682, 2000. 